\title{
Jesus Method to Compute the Circumference of A Circle and Exact $\pi$ Value
}

\author{
RD Sarava Jagannada Reddy
}

\section{Introduction}

The Holy Bible has said $\pi$ value is equal to 3 . Mathematicians were not satisfied with the value. They thought over. Pythagorean theorem came in the mean time. A regular polygon with known perimeter was inscribed in a circle and the sides doubled successively until the inscribed polygon touches the circumference, leaving no gap between them. Hence this method is called Exhaustion method. The value of the perimeter of the inscribed polygon is calculated applying Pythagorean theorem and is attributed to the circumference of the circle. This method was interpreted, first time, on scientific lines by Archimedes of Syracuse, Greece. He has said $\pi$ value is less than $31 / 7$.

Later mathematicians have refined the Exhaustion method and found many decimals. The value is $3.1415926 \ldots$ and this value has been made official.

From $15^{\text {th }}$ century (Madhava (1450) of South India) onwards infinite series has been used for more decimals to compute 3.1415926 of geometrical method. Notable people are Francois Viete (1579), Van Ceulen (1596), John Wallis (1655), William Brouncker (1658) James Gregory (1660), G.W. Leibnitz (1658), Isaac Newton (1666), Machin (1776), Euler (1748), S. Ramanujan (1914), Chudnovsky brothers (1989). The latest infinite series for the computation of $\pi$ value is that of David Bailey, Peter Borwein and Simon Plouffe (1996) and is as follows:

$$
\pi=\sum_{i=0}^{\alpha} \frac{1}{16^{i}}\left(\frac{4}{8 i+1}-\frac{2}{8 i+4}-\frac{1}{8 i+5}-\frac{1}{8 i+6}\right)
$$

Using above formula Yasumasa Kanada of Tokyo University, Japan, calculated trillions of decimals to $3.1415926 \ldots .$. with the help of super computer.

Mathematics is an exact science. We have compromised with an approximate value. Hence, many have tried to find exact $\pi$ value. This author is one among the millions. What is $\pi$ ? It is the ratio of circumference of a circle to its diameter. However, in Exhaustion method, perimeter of the inscribed polygon is divided by the diameter of the outside circle. Thus $3.1415926 \ldots$ violates the definition of $\pi$. This is about the value of $\pi$. Next, about the nature of $\pi$. C.L.F. Lindemann (1882) has said $\pi$ is a transcendental number based on Euler's formula $e^{i \pi}+1=0$. In Mathematical Cranks, Underwood Dudley has said " $\pi$ 's only position in mathematics is its relation to infinite services (and) that $\pi$ has no relation to the circle.... Lindemann proclaimed the squaring of the circle impossible, but Lindemann's proof is misleading for he uses numbers (which are approximate in themselves) in his proof".

Hence, pre-infinite series - days of geometrical method is approached again to find out exact $\pi$ value and squaring of circle. This author has struggled for 26 years (1972 to 1998) and calculated the exact value of $\pi$ in March, 1998. The following method calculates the total length of circumference and thus the exact $\pi$ value has been derived from it.

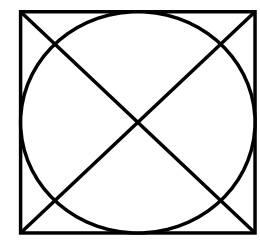

Procedure: Draw a square. Draw two diagonals. Inscribe a circle. Side $=a$, Diagonal $=\sqrt{2} a$, Diameter is also $=\mathrm{a}=\mathrm{d}$.

1) Straighten the square. Perimeter $=4 \mathrm{a}$

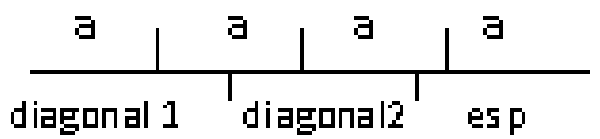

Perimeter - Sum of the lengths of two diagonals $=4 a-2 \sqrt{2} a=$ esp esp $=$ end segment of the perimeter of the square. 
2) Straighten similarly the circumference of the inscribed circle

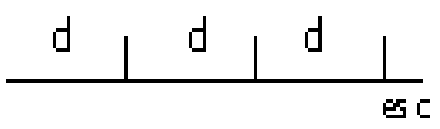

3 diameters plus some length, is equal to the length of the circumference.

Let us say circumference $=\mathrm{x}$.

Circumference -3 diameters $=\mathrm{x}-3 \mathrm{a}=\mathrm{esc}$ esc $=$ end segment of the circumference of the circle.

3) When the side of the square is equal to ' $a$ ', the radius of the inscribed circle is equal to $a / 2$. So, the radius is $1 / 8^{\text {th }}$ of the perimeter of the square.

4) The above relation also exists between the end segment of the circumference of the circle and the end segment of the perimeter of the square.

Thus as radius $\left(\frac{a}{2}\right)$ of the inscribed circle is to the perimeter of the square (4a), i.e., $1 / \mathbf{s}^{\text {th }}$ of it, so also, is the end segment of the circumference of the circle, to the end segment of the perimeter of the square.

So, the end segment of the circumference $=\frac{\text { end segment of the perimeter of the square }}{8}$

$$
\text { esc }=\frac{e s p}{8}=x-3 a=\frac{4 a-2 \sqrt{2} a}{8} \quad \therefore x=\frac{14 a-\sqrt{2} a}{4}
$$

5) Circumference of the circle $=\pi \mathrm{d}=\pi \mathrm{a}$ (where $\mathrm{a}=\mathrm{d}=$ diameter)

$$
\pi a=\frac{14 a-\sqrt{2} a}{4} \quad \therefore \pi=\frac{14-\sqrt{2}}{4}
$$

\section{Conclusion}

$\pi$ value, derived from the Jesus proof is algebraic, being a root of $x^{2}-56 x+97=0$ but also that it differs from the usually accepted value in the third decimal place, being $3.146 \ldots .$. 